\title{
An Instrumented Shoe for Ambulatory Prevention of Diabetic Foot Ulceration
}

\author{
Anwar S. Benbakhti, Samir Boukhenous, Cherif Zizoua and Mokhtar Attari \\ Laboratory of Instrumentation, LINS, Faculty of Electronics and Computers \\ USTHB, BP.32, El-Alia, 16111 Bab-Ezzouar, Algiers, ALGERIA \\ anwar.benbakhti@gmail.com, sboukhenous@gmail.com, zizoua.cherif@gmail.com, attari.mo@gmail.com
}

\begin{abstract}
The diabetic foot may present many complications if it is not regularly controlled. Statistics show that $25 \%$ of diabetics that develop peripheral neuropathy will develop a foot ulcer, more than half of them will become infected and $80 \%$ require non-traumatic amputation. This paper presents the instrumentation of an ordinary shoe for the monitoring of diabetic feet to reduce the risk of ulcers. The system contains an insole pressure distribution sensor to monitor the pressure in high risk ulcer points. It also contains temperature sensors placed in critical contact area to give feedback of the friction rate, and a humidity sensor to give feedback of the sweating rate. Since the aim of the system is to give a diabetic person the ability to monitor those critical points, the different sensors communicate wirelessly through Bluetooth with an Android-based phone application.
\end{abstract}

Keywords - diabetic foot, instrumented shoe, plantar pressure

\section{INTRODUCTION}

Diabetic foot ulcer increases every year, mainly because of the lack of information on the pathology of the diabetic foot and how to prevent it [1]. Even if a diabetic person's feet is monitored every day, the risk is still high due to the difficulty to detect early signs of an ulcer. Unfortunately, 15 percent of diabetic people will have a foot ulcer in their life, and 24 percent of them will require a lower extremity amputation. This makes diabetes the leading cause of amputation of the lower limb [2]. For these reasons, the importance of identifying individuals at risk is critical for eventual prevention. A trained staff (doctor, nurse, etc.), is assigned to the task of identifying and evaluating people at risk, yearly for people with low risk and monthly for those with high risk [1]. Sadly, not every doctor inspects the patient's feet at every visit, and not every diabetic person respects a normal visiting schedule. The case get worst especially when the patients lack the skills to perform a visual inspection of themselves.

The main reason of this trauma is a peripheral artery disease caused by poor blood flow and nerve damage caused by excessive glucose in the diabetic blood [3]. This results in a loss of sense in the sole, which leads to a poor or inexistent feeling of pain, heat or cold in the lower limb. The feet may not feel a fold in the socks, an object in the shoes or any other thing, provoking an excessive friction between the skin and the sock or shoes, which increases the risk of infections and ulceration.

The type and quality of shoes are critical to ensure a healthy diabetic foot. A bad environment (high humidity and temperature, bad pressure distribution due to the quality of the shoe, high friction areas between the skin/sock and the shoe ...) can affect the gait [4], the shoes must therefore be taken into account and be chosen carefully according to the physiology of the foot [5]. Unfortunately, the shoes available in the market have standardized shapes that do not match every foot shape. Bad quality shoes may cause corn and callus, too much rubbing on high-pressure area, thus increasing the risk of feet infection. Blisters can also be caused by excessive friction and temperature elevation. Their size increases with humidity, which also increases the risk to damage and exposes them to infection [6].

In addition to the classical foot examinations done yearly by a doctor for a normal low risk diabetic foot, the patient could benefit from a self-monitoring device to have a real time feedback about his feet environment. Fig. 1 shows an example of an ulcer caused probably by a bad shoe.

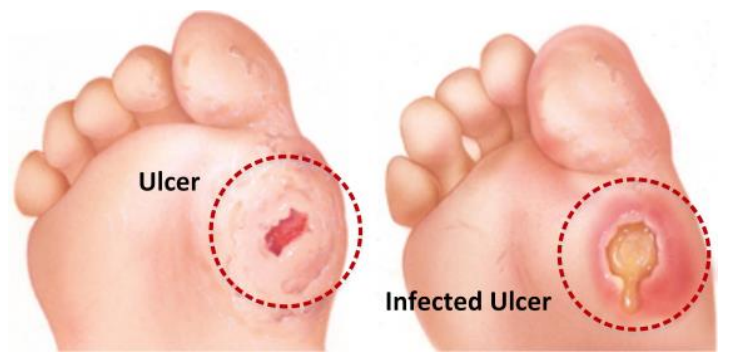

Fig. 1. Feet with ulcer

Taketoshi Mori et al demonstrate [4] the importance of the plantar pressure and of shear force measurement in the prevention of diabetic ulcer and the effect of the presence of callus in a patient's foot. SHU Lin et al. [7] developed a footwear system for diabetic patients capable of measuring and recording spatial and temporal pressure distribution, temperature, centre of pressure and accelerations for daily activities. A. Perrier et al. [8] developed washable socks for diabetic foot prevention using wireless sensors, which communicate through phone. Many other papers [9], [10] and [11] talk about the importance of these parameters in the diabetic foot ulcer prevention, mainly the analysis quantification of the plantar pressure repartition during the different phases of the gait.

The aim of our system is to give a real-time active feedback data to prevent the risk of ulceration for a diabetic person. It consists in an ordinary shoe with a foot pressure distribution sensor embedded in the insole, a coupled moisture/temperature sensor and three temperature sensors situated in high friction areas. The pressure distribution system consists in an insole 
developed in laboratory; it contains six Force Sensing Resistors (FSR). The temperature sensors are located in the high-risk area and the moisture sensor is placed under the sole and records the humidity through a small hole between the sensor and the skin.

The sensors' data are sent to an Android-based phone through Bluetooth. The Android application is of easy use and contains the various data and medical recommendations. Alerts will be set according to the sensors' values to inform the user about an eventual risk of disease caused by these parameters.

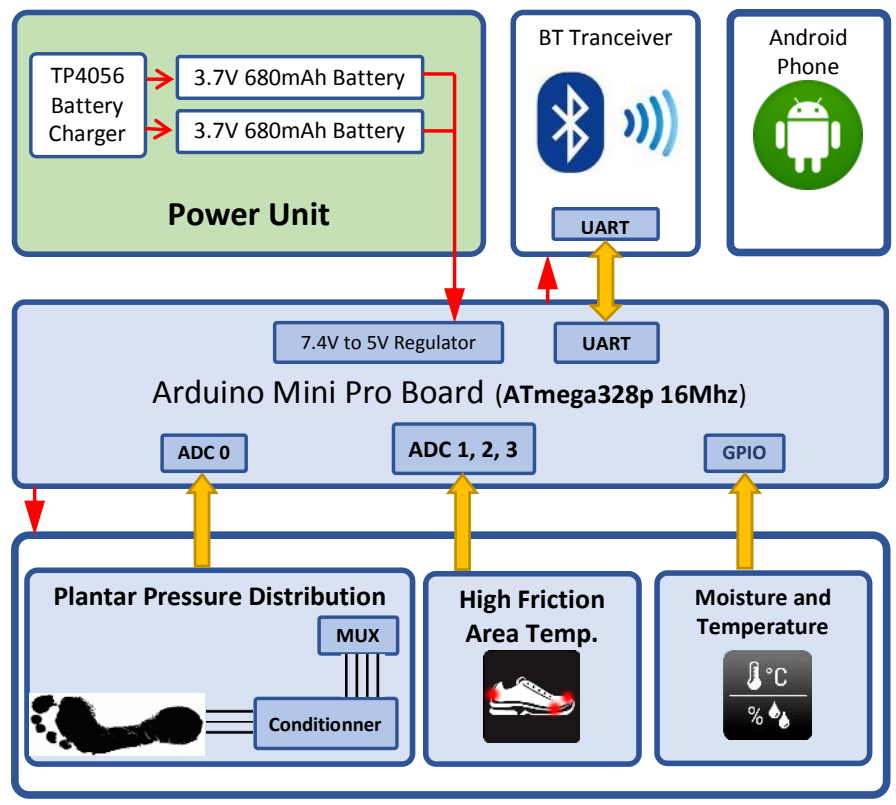

Fig. 2. System architecture

\section{SYSTEM DESCRIPTION}

\section{A. The foot plantar pressure distribution}

Bad pressure distribution is the main cause of foot ulcer in diabetic persons. Thus, the first parameter to monitor is the plantar pressure. Many sensors can be used for this aim such as piezoelectric sensors, load cells, strain gages and FSR. The latter was chosen for this purpose [5], as it is an easy to use technique based on a polymer sensing film whose resistance decreases with the applied pressure. Each sensor measures the dynamic weight in a specified point and displays the result using a colour indication in a Graphic User Interface (GUI) custom Android application.

The sensors used to build the insole are the 402 FSR from Interlink Electronics with a diameter of $18.3 \mathrm{~mm}$ and an active sensing area of $12.7 \mathrm{~mm}$. Flexibility, size and ease of use were the criteria of choice as it is mainly used in biomechanics to map and monitor foot pressure distribution [12].

To maximize the accuracy of the results, a uniform force distribution on the active area has to be provided [13]. For this end, a rigid coating dome made of epoxy and metal was used. The dome is glued to the FSR using a thin double-sided tape. This shape allows us to distribute a pressure from a little surface to the entire active area of the sensor. This form also prevents the sensor from being saturated with a punctual applied pressure and from exceeding the current density of $1 \mathrm{~mA} / \mathrm{cm}^{2}$ recommended by the manufacturer [14].

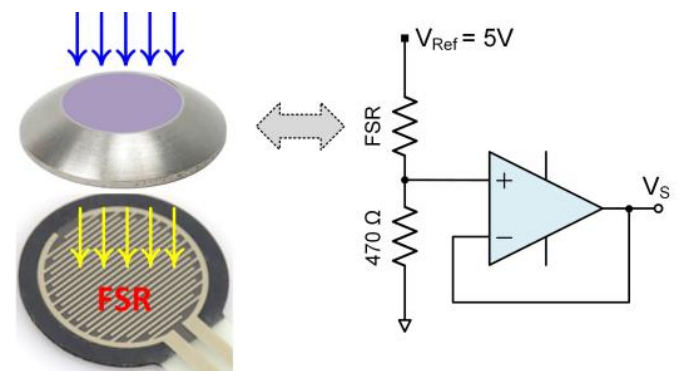

Fig. 3. Applied force and it distribution on the active area with the conditioner circuit

The FSRs are conditioned using a voltage divider (configured for a person with an average weight of $60 \mathrm{~kg}$ ) and followed by an Op Amp connected as follower to achieve impedance matching with the microcontroller. All outputs are connected to a Multiplexer, and then fed to the Analog/Digital Converter unit of an ATmega328p microcontroller mounted on an Arduino Mini Pro Board. The digitalized data are sent to an Android phone through an HC-06 Bluetooth module using the Serial Port Profile (SPP) and processed according to the calibration results.

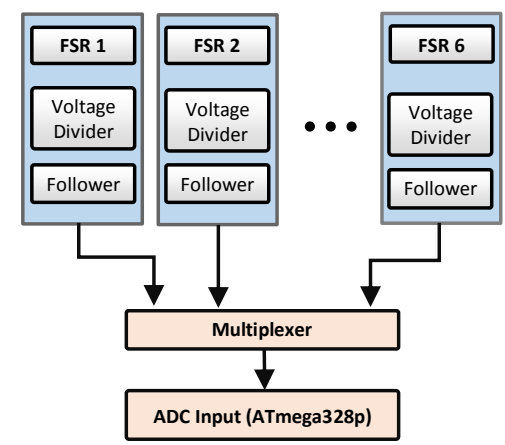

Fig. 4. Architecture of the FSR data acquisition sweeping

The area of high pressure was chosen according to a study where 87 patients had 103 existing foot ulcers [16], so the FSR was placed in the original insole perforated according to 1: Toe (T), 2: Medial Forefoot (MF), 3: Lateral Forefoot (LF), 4: Medial Med-foot (MM), 5: Lateral Mid-foot (LM) and 6: Heel (H) as shown in Fig 3. Finally, a third thin and supple layer was added to avoid any uncomfortable sensations.

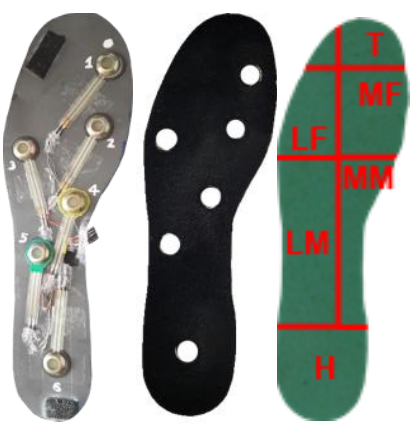

Fig. 5. Plantar pressure distribution insole 


\section{B. Temperature of high friction area}

As temperature is an important parameter that affects the skin condition, we placed three temperature sensors in areas where the friction between the skin and the shoe is the highest. These frictions can cause skin damage and may end with injuries that increase the risk of ulcer. Studies have shown that a difference of $2.2^{\circ} \mathrm{C}$ between two risky areas may indicate an ulcer [16].

Our system uses TMP35 temperature sensors placed under a layer of fabric present in the shoes. These sensors are placed so that the patient will not feel them.

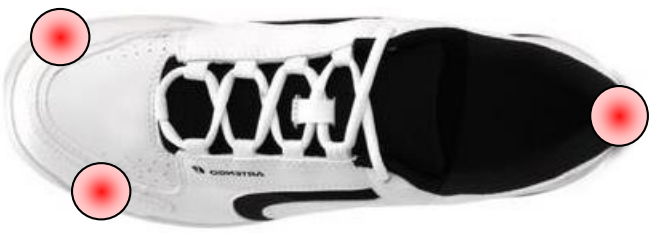

Fig. 6. High friction areas

\section{Humidity and temperature of the shoe}

In addition to the three temperature sensing points, we placed a coupled Temperature/Humidity SHT1X sensor under the insole. Humidity plays an important role in the skin condition and is a useful foot parameter for research, diagnosis and evaluation of human physiological condition [7]. In general, footwear prevents sweat from evaporating. Socks may also act as a sponge and lock moisture in. This can become dangerous because this environment is suitable for bacterial development and increases the risk of infection in the case of skin injury. For these reasons, the system collects the humidity rate and gives an alert if the humidity exceeds a certain rate during a predefined time set by a trained staff or the patient himself.

\section{Central unit}

All sensors are connected to the Arduino Mini pro board through a motherboard. The board contains two LM324 chips (each one containing four Operational Amplifiers), 4051 multiplexer to connect the six FSR to the ADC input and the HC-06, SHT1x, TMP35 connections.

Two Li-Ion batteries provide the power supply with a TP4056 charge controller. We added a connection to select between two modes, the first mode connect the batteries in parallel in order to be charged and the second mode connect the batteries in series to supply the regulator mounted on the Arduino Board.

\section{E. The Android application}

The aim of our system is to be accessible by everyone. For this, we chose to use an Android-based phone as the Human-toMachine interface for its popularity and simplicity of use. The developed application is structured into four screens with a "Main Menu" to switch between them. The first screen deals with the temperature and the humidity of the shoe collected by the SHT1X sensor. It displays the data and gives an alert if a predefined threshold is exceeded. The second screen gives the six plantar pressure distribution points collected by the FSRs. The pressures are represented by a colour variation from blue for a low load weight to red for a maximum load. The data is also stored in a text file to be accessed for a better analysis. An alert is given if a dangerous pressure is detected for a prolonged time. The third screen provides information about the temperature of high friction areas. Finally, the fourth screen connects the application to a website to give recommendations and advice from professional doctors of different fields concerning diabetes and the way to prevent complications.

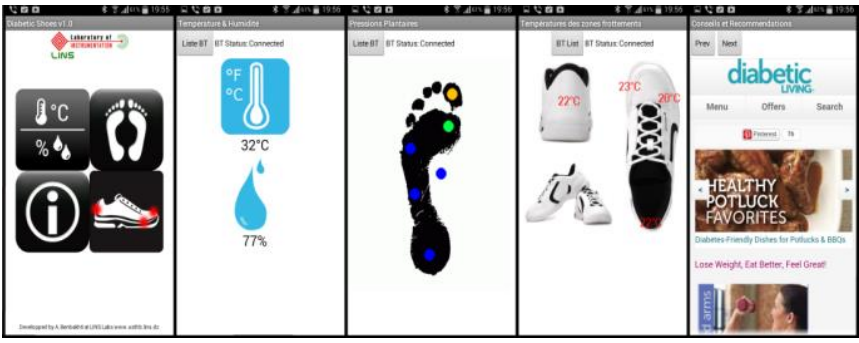

Fig. 8. The android application

\section{SYSTEM CALIBRATION}

The temperature and humidity sensors (TMP35 and SHT1X) are factory calibrated, but FSRs need calibration. This is due to the polymer in which the FSR is made, and due to the dome added on the active area which change the sensors' response, so each FSR need a calibration. Fig. 9 gives four examples of FSR's outputs voltages according to different applied forces.
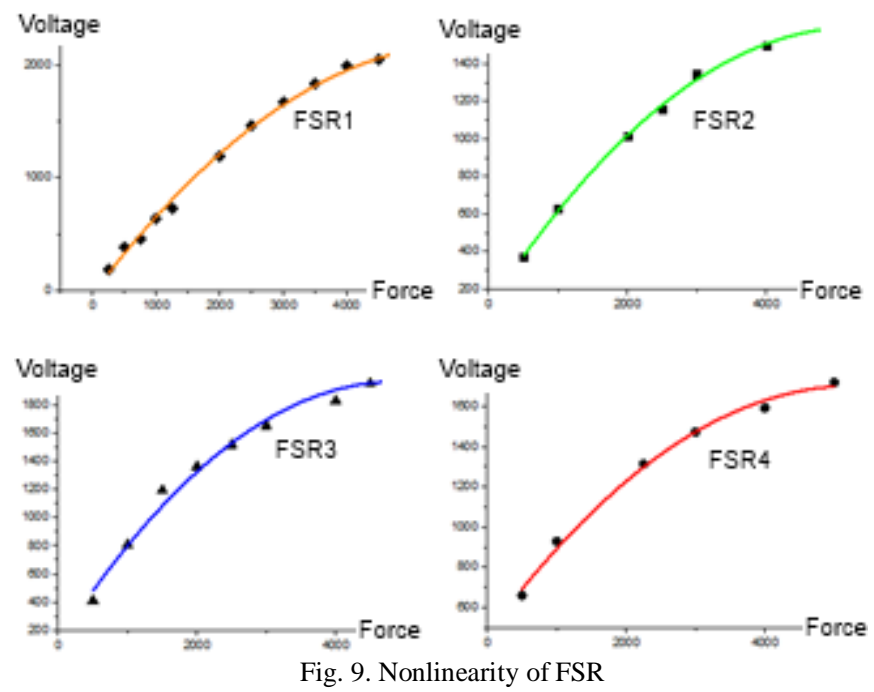

These graphs show that the FSRs are nonlinear over the whole range and each FSR gives different results. A fourth degree polynomial function was calculated for each FSR to approximate the data, thus:

$$
F(V)=b_{0}+b_{1} V+b_{2} V^{2}+b_{3} V^{3}+b_{4} V^{4}
$$

Where $F$ is the force applied on the FSR and $V$ the voltage output of the conditioning circuit, $b_{x}$ are the coefficients of the polynomial. These ones are implemented into the Android application because of the greater calculation speed of smartphone processors compared to that of an ATmega328p microcontroller. 


\section{TESTS AND RESUlTS}

To demonstrate the feasibility of the system and give an example of the results, we performed a test on a healthy person. The data was collected and recorded by the foot pressure distribution insole during 10 tests, as shown in Fig. 11.
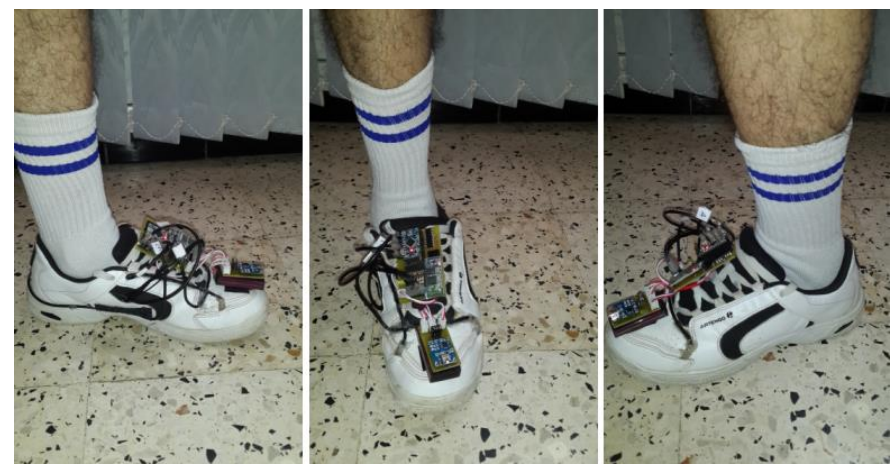

Fig. 10. Foot wearing the footwear system

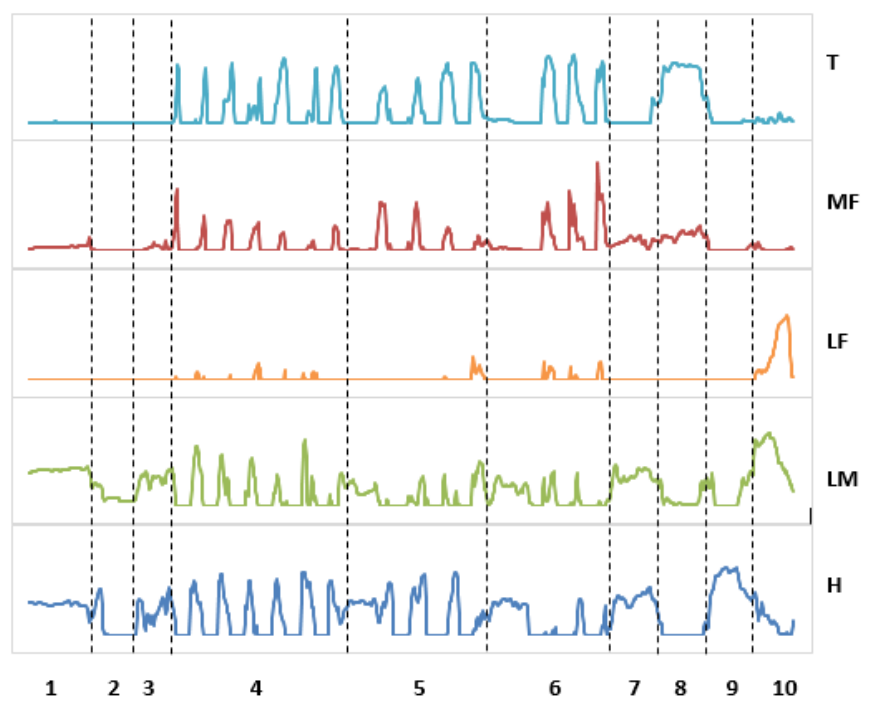

Fig. 11. Tests of the plantar pressure distribution

1: Stand-up, 2: Sit-down, 3: Stand-up, 4: Walk 6 steps forward, 5: Walk 3 steps backward, 6: Walk 3 steps leftward, 7: Stand, 8: Lean forward, 9: Lean backward, 10: Lean Leftward.

The risk of ulceration is not the same for every individual, and depends on the anatomical properties of the patient's foot (bones curvature, thickness of the soft tissues, etc.) [18], the system's alerts must be tuned by a trained staff. We performed only virtual alerts for random values to test the system and no trial on diabetic person was performed yet.

\section{CONCLUSION}

An ordinary shoe equipped with specified sensors has been developed and transformed into an intelligent footwear in order to prevent ulcer formation in diabetic persons by providing feedback about the various physical parameters in the shoe and gives alerts if anomalies are detected. Tests on healthy persons were carried out to demonstrate the feasibility of the system. For future work, tests during a long period has to be done on diabetic persons in different situations to monitor the evolution of the parameters. The system is a laboratory prototype, so a smaller version could be a better solution to minimize discomfort during gait. The phone application will include a special page for the trained staff to set the threshold's alerts. Storing the data in a web database and sharing them with a trained staff can gives to the system a telemedicine and remote capability.

\section{REFERENCES}

[1] C. Tobin, D. Armstrong, R. Frykberg, C. kennedy, M. Parrot, P. Colman, Feet can last a lifetime, A health care provider's guide to preventing diabetes foot problems, National Diabetes Education Program (NDEP). Nov 2000.

[2] N. Singh, D. Armstrong, and B. Lipsky, "Preventing foot ulcers in patients with diabetes," JAMA: the journal of the American Medical Association, vol. 293, no. 2, pp. 217-228, 2005.

[3] R. Macfarlane and W. Jeffcoate, "Factors contributing to the presentation of diabetic foot ulcers," Diabetic medicine, vol. 14, no. 10, pp. 867-870, 1997.

[4] T. Mori, M. Hamatani, H. Noguchi, M. Oe and S. Sanada, "Insole-Type simultaneous measurement sysem of plantar pressure and shear force during gait for diabetic patients", Journal of Robotics and Mechatronics, vol. 24, no 5, pp. 776-772, 2012.

[5] S. Ostadabbas, A, Saeed, M. Nourani, M. Pompeo, "Sensor architectural tradeoff for diabetic foot ulcer monitoring", $34^{\text {th }}$ Annual International Conference of the IEEE EMBS, San Diego, California USA, 28 August 1 September 2012.

[6] NIDDKD Institute, "Prevent diabetes problems: Keep your feet healthy", Guide from the National Institute of Diabetes and Digestive and Kidney Diseases. No. 14-4282; Feb 2014.

[7] Shu Lin et all, "Monitoring diabetic patients by novel intelligent footwear system" International Conference on Computerized Healthcare (ICCH), in M. Young Ed, The Technical Writer's Handbook. Mill Valley, Vol., $\mathrm{N}^{\circ}$., pp.91-94, December 2012

[8] A. Perrier, N. Vuillerme, V. Luboz, M. Bucki, F. Cannard, B. Diot, D. Colin, D. Rin, J.-P. Bourg, Y. Payan, "Smart diabetic socks: embedded device for diabetic foot prevention", IRBM, Vol 35, no 2, pp. 72-76, Apr 2014.

[9] M Lord, R Hosein, "A study of in-shoe plantar shear in patients with diabetic neuropathy," Clinical Biomechanics, Vol. 15, $\mathrm{N}^{\circ} 4$, pp. 278-283, May 2000.

[10] F. Dabiri et all., "Electronic orthotics shoe: Preventing ulceration in diabetic patients," Engineering in Medicine and Biology Society, EMBS 2008. 30th Annual International Conference of the IEEE, Vol., $\mathrm{N}^{\circ}$., pp.771-774, 20-25 Aug. 2008M. Young, The Technical Writer's Handbook. Mill Valley.

[11] Bacarin TA, Sacco IC, Hennig EM. "Plantar pressure distribution patterns during gait in diabetic neuropathy patients with a history of foot ulcers," Clinics (Sao Paulo). Vol. 64, no. 2, pp. 113-120, 2009

[12] A. H. Abdul Razak, A. Zayegh, A. K. Begg and Y. Wahab, "Foot plantar pressure measurement system: a review," Sensors, n. 12, 2012.

[13] Florez, J.A.; Velasquez, A., 'Calibration of force sensing resistors (fsr) for static and dynamic applications,' ANDESCON, 2010 IEEE, vol., no., pp.1,6, 15-17 Sept. 2010.

[14] Interlink Electronics Inc., FSR Force Sensing Resistor Integration Guide and Evaluation Parts Catalog, Interlink Electronics, 2014.

[15] L. Lavery, E. Peters, and D. Armstrong, "What are the most effective interventions in preventing diabetic foot ulcers," International Wound Journal, Vol. 5, $\mathrm{N}^{\circ}$ 3, pp. 425-433, 2008.

[16] R. G. Fryberg, L. A. Lavery, H. Pham, C. Harvey, L. Harkless, A. Veves, "Role of neuropathy and high foot pressures in diabetic foot ulceration," Diabetes Care, vol. 21, no. 10, Oct, 1998.

[17] A. Perrier, N. Vuillerme, V. Luboz, M. Bucki, F. Cannard, B. Diot, D. Colin, D. Rin, J.-P. Bourg, Y. Payan, "Smart Diabetic Socks: Embedded device for diabetic foot prevention,' IRBM, Volume 35, Issue 2, April 2014, Pages 72-76. 